\title{
Quality and best practises
}

\author{
Henk ten Have ${ }^{1}$
}

Published online: 22 February 2017

(C) Springer International Publishing AG 2017

In ethics education there is an abundant variety of activities. Educational approaches and efforts differ in almost all dimensions. Sometimes it will be difficult to identify any common approaches and standards. Nonetheless, not all programs and efforts have the same level and quality. Intuitively we may distinguish excellent educational activities from less successful ones. We will all remember inspiring ethics teachers, and challenging and superb programs, but we also have bad experiences with weak and inadequate programs and scholars. But what exactly is quality of ethics education?

This issue of the journal starts with a study of Ercan Avci. He observes that the issue of quality in ethics education will require the determination of certain common indicators based on standards and principles in ethics education. However before we can do that, the current situation in ethics teaching programs should be examined in order to detect good practices as well as shortcomings of ethics teaching in connection to its aims, scope, and methods. In his paper, Avci reviewed the literature on ethics education (Avci 2017). His findings demonstrate that both students and educators believe that ethics education generates positive impact on ethical awareness, knowledge, and reasoning. They also clarify that not many conclusions can be drawn concerning quality in ethics education.

Similar conclusions result from the second publication in this issue. John Stone and colleagues review the growing literature about graduate online bioethics programs (Stone et al. 2017). Online teaching programs are mushrooming, also in the area of bioethics. Experiences are recent, and the body of literature reflecting on experiences is limited. Stone tries to determine on the basis of existing literature, but also his own experiences with these online programs at Creighton University, whether any best practices can be identified. Unfortunately, this is not possible; all practices and experiences are provisional.

Many people will possibly wonder about this question of quality. The notion is not clear, and it is not evident to apply it in the field of ethics teaching. Maybe it applies to

Henk ten Have

tenhaveh@duq.edu

1 McAnulty College and Graduate School of Liberal Arts, Center for Healthcare Ethics, Duquesne University, Fisher Hall 300, 600 Forbes Avenue, Pittsburgh, PA 15282, USA 
teaching but how and why is it connected to ethics? On the other hand, in the field of healthcare, the notion of quality is popular. Perhaps its use in this field can give some inspiration for ethics teaching.

In order to elucidate the notion of quality in the context of ethics education, lessons can be learned from the development of studies in quality of care. In this relatively new discipline of care studies, in order to determine what good care is, a distinction is made between structure, process, and outcome. Another, related approach is to distinguish three perspectives to define, assess and improve quality of care. The user perspective emphasizes satisfaction and outcome; it is focused on expectations and preferences (patient demands); it takes into account dimensions such as support, information, and choice. The provider perspective emphasizes professional standards and guidelines; it focuses on needs as defined by professionals; it takes into account dimensions such as effectiveness, accessibility, continuity, technical competence, and personal attention. The policy perspective emphasizes the system and organization of care; it focuses on the context in which care is delivered; it takes into account dimensions such as availability, efficiency, and organization. Quality of care is the balance between these three perspectives.

The experiences in quality of care can be helpful to further the debate on quality in ethics education. Using the Donabedian model, a more detailed framework of relevant dimensions can be constructed. Each of these dimensions can be refined from the three perspectives of students (users), teachers (providers) and organizations (policy-makers in universities, hospitals and professional bodies). They emphasize respectively perception, performance, and effectiveness. From the student perspective, teaching methods should aim at interaction, communication, and deliberation. The teacher perspective might articulate integration, while the policy perspective emphasizes accountability. Different perspectives might also agree, for example on the priority of case-based and problem-based learning.

When the relevant dimensions of quality are identified, steps can be made to enhance the quality of ethics education. Like in the field of quality of care, various activities can then be undertaken: development of standards, guidelines and arrangements for quality ethics education; documenting and assessing ethics education activities; analyzing the discrepancy between what is desired and what is achieved; assuring and improving the quality of ethics education, i.e. improving the balance between desired and achieved quality.

\section{References}

Avci, Ercan. 2017. Learning from experiences to determine quality in ethics education. International Journal of Ethics Education 2(1). in this issue.

Stone, John R., Helen Stanton Chapple, Amy Haddad, Sarah Lux, and Christy A. Rentmeester. 2017. Discussion in graduate online bioethics programs International Journal of Ethics Education 2(1). in this issue. 